with fixed line length and fixed block size in 7 track, BCD, 200,556 or $800 \mathrm{CPI}$; or 9 track, ASCII or EBCDIC, 800 or $1600 \mathrm{CPI}$; or punched cards with IBM 026 or IBM 029 codes; or paper tape, 8-level ASCII are equally acceptable machine-readable media for deposition. Each deposition should be accompanied by sufficient information to allow ready transcription of the medium, such as a FORMAT statement and a designation of the recording equipment used.

Acta Cryst. (1981). B37, 1162

\section{Commission on Journals}

\section{Submission of Manuscripts Based on Powder Diffraction Profile Fitting or Refinement (Rietveld) Methods: Deposition of Data}

A steadily increasing number of manuscripts that depend on the use of either powder diffraction profile fitting or refinement (Rietveld) methods are being submitted for publication. Commission policy has recently required that figures in such manuscripts that present the experimental and calculated diffraction profiles of the material studied should also contain the difference profile $\left(I_{\mathrm{obs}}-I_{\text {calc }}\right)$, as an aid to the reader. It is recognized that the primary diffraction data cannot be extracted satisfactorily from such figures. The Commission has now decided that, in addition to the figure, the authors of such manuscripts should deposit the numerical intensity of each measured point on the profile, as a function of scattering angle.
The attention of authors is also drawn to notices concerning stereofigures | Acta Cry'st. (1978). B34, 3846], dimensions of material for deposition lActa Cry'st. (1979). B35, 7921, estimated standard deviations, SI units and anisotropic thermal parameters |Acta Cry'st. (1979). B35, 1302], submission of connected computer output | Acta Crust. (1979). B35, 2284-2285l, chemical-connectivity relationships | Acta Cry'st. (1980). B36, 1524|, and estimated standard deviations with a zero value for varied parameters [Acta Cryst. (1980). B36, 2508], in addition to the information given in Notes for Authors | Acta Cry'st. (1978). A34, 143-157l.

Acta Cryst. (1981). B37, 1162

\section{Communicated Abstracts}

\section{Twelfth International Congress of Crystallography Carleton University, Ottawa 16-25 August 1981}

The abstracts of papers communicated to the Congress will be published as a Supplement to Acta Crystallographica, Section A. The Supplement will contain abstracts directly reproduced from typescript copy furnished by the authors. Copies will be sent gratis to subscribers to Section A, but not to subscribers to Section B or to Journal of Applied Crystallography. However, copies may be ordered direct from the publishers, Munksgaard, 35 Nørre Søgade, DK-1370 Copenhagen K. Denmark, at a price of 170 Danish kroner. 